Vol 15 lss 4

May 2018
A Publication of the Systems and Psychosocial Advances Research Center

A Massachusetts Department of Mental Health Research Center of Excellence

\title{
Detecting and Intervening on Suicidality in Emergency Departments: The ED-SAFE Study
}

Edwin Boudreaux, Carlos A. Camargo, Ivan Miller, and the ED-SAFE investigators

\section{Suicidality in Emergency Departments}

In 2016, there were 44,695 deaths by suicide in the United States. ${ }^{1}$ Suicide accounts for $1.6 \%$ of all deaths and is the tenth leading cause of death in the United States. ${ }^{1}$ Suicide attempts are much more common, with more than 1 million people per year attempting suicide.

Healthcare settings can play a vital role in screening patients for suicide risk and providing appropriate intervention and referral to treatment. Many individuals at risk for suicide are seen at emergency departments (EDs), often presenting with chief complaints that are not suicide-related. As a result, an ED visit can represent a unique opportunity for suicide risk screening and intervention; yet ED-initiated suicide prevention efforts remain underdeveloped. At the time this study was funded, there were no suicide risk screeners validated for primary detection among adult ED patients. In order to prevent suicide it is critical to develop and test feasible approaches to universal ED-based screening for suicide risk, as well as effective interventions that can be initiated at the ED visit.

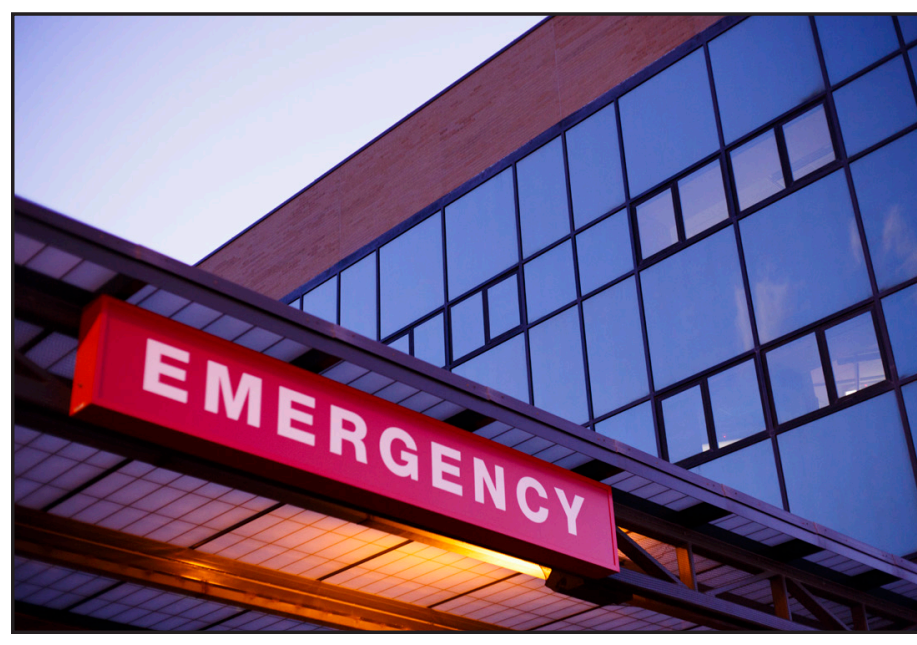

\section{Detecting Suicidality}

Adult ED patients with any presence of self-harm ideation or behavior documented on their medical charts were assessed for eligibility. ED patients were enrolled in the study if they confirmed either a suicide attempt or active suicidal ideation within the past week and agreed to study requirements.

ED-SAFE enrolled 1,376 participants, the majority of

\section{ED-SAFE Study Goals}

In response to this critical need in 2009, the National Institute for Mental Health funded ED Safety Assessment and Follow-up Evaluation (ED-SAFE). ED-SAFE was a large, three-phase suicide intervention trial designed to determine if an ED-initiated intervention could reduce subsequent suicidal behavior.

\section{The trial had two goals:}

1. Develop and test a standardized approach to universal suicide risk screening within EDs (the Screening Evaluation); and

2. Test an ED-initiated intervention with follow-up telephone contact to reduce suicidal behavior among individuals who screened positive for suicide risk in the ED (the Intervention Evaluation). 
whom were female and non-Hispanic. The median age was 37 years old. One-third of participants had made a suicide attempt in the week prior to the ED visit, $87 \%$ had a psychiatric disorder, and almost $70 \%$ had a coexisting medical disorder. Participants enrolled in the prospective portion of the study were followed for one year using a telephone assessment and medical record review.

ED-SAFE consisted of three sequential research phases: Treatment as Usual (Phase 1), Universal Screening (Phase 2), and Universal Screening + Intervention (Phase 3). ED-SAFE was conducted at 8 hospital EDs, ranging from small community hospitals to large academic centers, across 7 states. Participating hospitals did not have dedicated psychiatric EDs.

The primary goal of Phase 1 was to provide baseline detection data. Over the course of first ten months of the study, research assistants at each of the sites prospectively screened ED charts for documentation of intentional self-harm. These patients were provided usual and customary screening care specific to the site, which enabled a determination of each site's natural rate of screening and detection of self-harm ideation and behavior.

In Phase 2, since there was no pre-existing, validated suicide screening instrument for universal screening in ED settings, the research team created and implemented the 3-item Patient Safety Screener (PSS-3). ${ }^{2}$ The first question assesses depressed mood, the second assesses active suicidal ideation, and the third assesses lifetime suicide attempt. The PSS-3 was completed by highly-trained primary treating nurses with all adults admitted to the ED. A positive screen was defined as active suicidal ideation in the past 2 weeks or a suicide attempt within the past 6 months. The PSS- 3 was validated in a subsequent study ${ }^{3}$ and demonstrated good convergence with the Beck Scale for Suicide Ideation (BSSI), which is a longer and wellestablished suicide rating scale. During this screening phase, the study team examined whether universal suicide risk screening was feasible and effective at improving suicide risk detection in the ED. They also wanted to determine the outcomes of implementing a mandatory suicide risk screening in EDs.

\section{Intervening with Suicidal ED Patients}

In Phase 3, all sites continued universal screening with the PSS-3 and went on to implement a 3-component intervention with all participants that was composed of:

1. A secondary suicide risk screening designed for ED physicians to evaluate suicide risk following an initial positive screen on the PSS- 3

The research term created the Patient Safety Secondary Screener to be used by ED physicians to evaluate suicide risk after an initial positive screen on the PSS-3. ${ }^{4}$ The Patient Safety Secondary Screener for physicians consists of a brief review of risk factors commonly associated with suicide. ${ }^{4}$ This screener assisted ED physicians to decide whether to consult with psychiatry about the participant's suicidality. ${ }^{4}$

2. The provision of a self-administered safety plan and information to patients by nursing staff

Any participant who screened positive on the PSS-3 was provided with outpatient suicide prevention resources at discharge: a personalized, self-administered safety plan (modeled after work by Stanley and Brown) to identify early warning signs for suicidal behavior; internal and external coping resources; and an outpatient mental health resource guide including community resources and hotline numbers. ${ }^{13}$

3. A series of telephone calls to the participant, with the optional involvement of their significant other, for 52 weeks following the index ED visit ${ }^{4}$

The study team utilized a modification of the Coping Long-Term with Attempted Suicide Program (CLASP-ED) [Miller, R34MH073625, R01AA015950], an adjunctive intervention that combines principles of case management, individual counseling, and family/significant other mobilization. The intervention included assessments of participants at $6,12,24,26$, and 52 weeks after discharge with brief 10-20 minute telephone calls. A significant other of the participant (with the participant's permission) received up to 4 phone calls. 
Calls were administered by trained mental health advisors (counselors and psychologists).

\section{The content of the calls focused on:}

$\checkmark$ Identifying suicide risk factors

$\checkmark$ Clarifying values and goals

$\checkmark$ Safety and future planning

$\checkmark$ Facilitating treatment engagement/adherence

$\checkmark$ Facilitating patient-significant other problem solving

The primary instrument used for assessing suicidal ideation and behavior during follow-up telephone assessment calls was the Columbia Suicide Severity Rating Scale (CSSRS). ${ }^{6}$ Outcomes were assessed through telephone assessment results and medical record review over a 1 year follow-up period, with the primary outcome variable being suicide attempts. State and national vital statistics registries were also reviewed to detect any participant deaths.

\section{Outcomes for Reducing Suicide}

This study showed that universal suicide risk screening in the ED is feasible and almost doubled suicide risk detection in this population. ${ }^{2}$ If these findings remain true when scaled, the public health impact could be tremendous, because identification of risk is the first and necessary step for preventing suicide.

ED-SAFE also demonstrated that a multifaceted intervention can reduce future suicidal behavior in ED patients at risk for suicide. Participants in Phase 3 had significantly fewer total suicide attempts $(5 \%$ absolute decrease) in the 12-month follow-up period compared to the participants in Phase 1. ${ }^{7}$ Participants in Phase 3 had $30 \%$ fewer total suicide attempts than participants in Phases $1 \& 2 .^{7}$ These results, consistent with other studies' results, demonstrate the utility of contact following discharge from emergency departments.

ED-SAFE is a valuable study to help establish the feasibility, effectiveness, and sustainability of a multi-component screening and intervention for suicide within general ED settings. ED-SAFE results have practical implications for the prevention of suicide moving forward.

\section{Universal Screening}

Since ED-SAFE concluded, universal screening for suicide risk in the ED has become a practice recommended by the Joint Commission Sentinel alert no.56. The Emergency Department Safety Assessment and Follow-up Evaluation 2 (ED-SAFE-2; PI: Boudreaux) was conducted at the same eight sites as the first ED-SAFE study; it examined both the sustainability of universal suicide risk screening and implementation of the Safety Planning Intervention into routine care. The ED-SAFE study informed the System of Safety (SOS; PIs: Boudreaux \& Kiefe) study currently underway in the UMass Memorial Health Care system. SOS is the first study to examine the effects of universal suicide screening, safety planning, care transitions and other Zero Suicide model elements across emergency department, inpatient and outpatient settings.

These research efforts will inform and likely accelerate the adoption of best practices for suicide prevention across diverse health care settings, potentially saving many patients' lives. 


\section{Resources}

* Patient Safety Screener (PSS-3):

http://emnet-usa.org/EDSAFE/materials/K PtSafetyScreen.pdf

* Patient Safety Secondary Screener:

http://emnet-usa.org/EDSAFE/materials/Patient\%20Safety\%20Screener secondary 5-18-12\%20FINAL.pdf

\section{* Other ED-SAFE Materials:}

http://emnet-usa.org/EDSAFE/edsafe.htm

\section{* Stanley and Brown Safety Plan:}

http://www.suicidesafetyplan.com

\section{References}

${ }^{1}$ Drapeau, C. W., \& McIntosh, J.L. American Association of Suicidology. USA suicide: 2016 official final data. Centers for Disease Control and Prevention, National Center for Injury Prevention and Control. Accessed April 4, 2018. http:// www.suicidology.org/Portals/14/docs/Resources/FactSheets/2016/2016datapgsv1b.pdf?ver=2018-01-15-211057-387

${ }^{2}$ Boudreaux, E. D., Camargo, C. A., Jr., Arias, S. A., Sullivan, A. F., Allen, M. H., Goldstein, A. B., ... Miller, I. W. (2016). Improving suicide risk screening and detection in the emergency department. American Journal of Preventive Medicine, 50(4), 445-453. doi:10.1016/j.amepre.2015.09.029

${ }^{3}$ Boudreaux, E. D., Jaques, M. L., Brady, K. M., Matson, A., \& Allen, M. H. (2015). The Patient Safety Screener: Validation of a brief suicide risk screener for emergency department settings. Archives of Suicide Research, 19(2), 151-160. doi:10.1 080/13811118.2015.1034604

${ }^{4}$ Boudreaux, E. D., Miller, I., Goldstein, A. B., Sullivan, A. F., Allen, M. H., Manton, A. P., . . Camargo, C. A. (2013). The Emergency Department Safety Assessment and Follow-up Evaluation (ED-SAFE): Method and design considerations. Contemporary Clinical Trials, 36(1), 14-24. doi:10.1016/j.cct.2013.05.008

${ }^{5}$ Stanley, B., \& Brown, G. K. (2012). Safety planning intervention: A brief intervention to mitigate suicide risk. Cognitive and Behavioral Practice, 19(2), 256-264.

${ }^{6}$ Posner, K., Brown, G. K., Stanley, B., Brent, D. A., Yershova, K. V., Oquendo, M. A., . . Mann, J. J. (2011). The ColumbiaSuicide Severity Rating Scale: Initial validity and internal consistency findings from three multisite studies with adolescents and adults. American Journal of Psychiatry, 168(12), 1266-1277. doi:10.1176/appi.ajp.2011.10111704

${ }^{7}$ Miller, I. W., Camargo, C. A., Jr., Arias, S. A., Sullivan, A. F., Allen, M. H., Goldstein, A. B., . . Boudreaux, E. D. (2017). Suicide prevention in an emergency department population: The ED-SAFE study. JAMA Psychiatry, 74(6), 563-570. doi:10.1001/jamapsychiatry.2017.0678 